Bru Luna, L. M., Martí-Vilar, M. y González-Sala, F. (2020). Revisión sistemática de intervenciones en prosocialidā y empatía en personas con TEA. Revista de Investigación Educativa, 38(2), 359-377.

DOI: http://dx.doi.org/10.6018/rie.395421

\title{
Revisión sistemática de intervenciones en prosocialidad y empatía en personas con TEA
}

\section{Systematic review of interventions in prosociality and empathy in people with ASD}

\author{
Lluna María Bru Luna*, Manuel Martí-Vilar* y Francisco González-Sala** \\ *Departamento de Psicología Básica. Facultad de Psicología. Universitat de València (España) \\ **Departamento de Psicología Evolutiva y de la Educación. Facultad de Psicología. Universitat de València (España)
}

\begin{abstract}
Resumen
El DSM-5 enmarca el trastorno del espectro autista (TEA) dentro de los trastornos del desarrollo neurológico, cuyo rasgo más característico son las alteraciones de la conducta social, que incluyen afectaciones en prosocialidad y empatía. El objetivo del presente trabajo es determinar la efectividad de las diferentes intervenciones dirigidas a mejorar la prosocialidad y la empatía en personas con TEA e identificar factores determinantes. Para ello, se ha llevado a cabo una revisión sistemática según las directrices PRISMA de los artículos encontrados en las bases de datos Web of Science, PubMed, ScienceDirect y Dialnet. Los resultados indican un incremento en las puntuaciones en prosocialidad y empática para las personas con TEA mediante diferentes técnicas de intervención, aunque parece ser que los factores determinantes son el grado de lenguaje receptivo y expresivo, las habilidades de comunicación no verbal, el razonamiento fluido, el CI y la frecuencia en la implementación.
\end{abstract}

Palabras clave: intervención; autismo; empatía; prosocial.

\begin{abstract}
The DSM-5 frames the autism spectrum disorder (ASD) within the neurodevelopmental disorders, whose most characteristic features are the alterations of social behavior. These include

Correspondencia: Manuel Martí-Vilar, Manuel.Marti-Vilar@uv.es, Universitat de València, av. Blasco Ibañez, 21. CP: 46.010-València.
\end{abstract}


affectations in prosociality and empathy. The objective of this study is to determine the effectiveness of the different interventions aimed at improving prosociality and empathy in people with ASD and identifying determining factors for it. To this end, a systematic review of the articles found in the Web of Science, PubMed, ScienceDirect and Dialnet databases has been carried out following the PRISMA guidelines. The results indicate that an increase in prosociality and empathy scores for people with ASD is possible through different intervention techniques, and it seems that the determining factors are the degree of receptive and expressive language, nonverbal communication skills, fluid reasoning, IQ and the frequency of implementation.

Keywords: intervention; autism; empathy; prosocial.

\section{Introducción}

El trastorno del espectro autista (TEA) se encuentra enmarcado según el DSM-5 dentro de los trastornos del desarrollo neurológico. Principalmente, el TEA se caracteriza por la presencia de déficits persistentes en la comunicación e interacción social, así como por patrones de comportamiento, intereses o actividades restrictivas o repetitivas, presentes ya en los primeros años del desarrollo del niño (Asociación Americana de Psiquiatría, 2014). Quizá uno de los rasgos más llamativos de este trastorno sean estas alteraciones en el área social, que son incluso más evidentes en las edades más tempranas (Belloch, Sandín y Ramos, 2009). La conducta prosocial engloba conceptos como empatía, cooperación, ayuda, altruismo, entre otros, y constituye un término más general para designar a toda conducta social positiva con o sin motivación altruista (Martínez, Martí-Vilar y Martínez, 2015). Así, el correcto desarrollo de esta área se traduce generalmente en unos niveles adecuados de autoestima, empatía y capacidad de resolución de problemas interpersonales que, a priori, se encuentran afectados en los sujetos con TEA.

Tal y como afirman Spinrad y Gal (2018), aunque muchas veces los aumentos en la prosocialidad no son un enfoque específico de los programas de intervención, a menudo muchos de ellos enfatizan en varios de los precursores importantes del comportamiento prosocial, como la empatía, la comprensión de las emociones propias y ajenas y la autorregulación. Todos estos programas, de orientación psicoeducativa, como apuntan Mulas et al. (2010) y Etchepareborda (2005) buscan potenciar una comunicación funcional, espontánea, desarrollando habilidades sociales, habilidades adaptativas, reducir conductas inadecuadas y disruptivas y desarrollar habilidades cognitivas y ejecutivas.

A pesar de la necesidad que existe en emplear este tipo de intervenciones en personas del colectivo TEA, el panorama que existe es muy distinto. En la literatura apenas se encuentran artículos que estudien aspectos de la prosocialidad y empatía en este tipo de población, y muchos de los resultados apuntados en estos trabajos son difíciles de generalizar, principalmente por presentar limitaciones importantes en el tamaño y en las características de la muestra, por falta de un grupo control y la falta de método de doble ciego. Con respecto a esto último, Smith et al. (2007) proponen un protocolo con cuatro fases de cara al diseño e implementación de intervenciones psicosociales en sujetos TEA. En primer lugar, resulta necesario realizar estudios iniciales acerca de la eficacia de una intervención o técnica concreta. En segunda instancia elaborar 
manuales de intervención y pruebas piloto con el objetivo de conocer la viabilidad a la hora de aplicar una intervención concreta. Posteriormente, recogen la necesidad de aplicar a gran escala la intervención, controlando las condiciones que rodean al estudio y midiendo los resultados obtenidos, con el objetivo de la efectividad. Por último, cabe evaluar la efectividad de la intervención en contextos comunitarios, a pesar de las dificultades que entraña este tipo de estudios dado que las condiciones de contextuales y de los investigadores pueden ser muy diferentes.

Por ello, en este marco, el objetivo del presente trabajo es determinar la efectividad de las diferentes intervenciones dirigidas a mejorar la prosocialidad y la empatía en personas con TEA, así como identificar posibles factores determinantes, mediante una revisión sistemática de la literatura científica.

\section{Metodología}

Este trabajo incluye una revisión sistemática de la literatura científica relacionada con las intervenciones en prosocialidad y empatía dirigidas a personas con TEA. Para su correcta elaboración, se han seguido las directrices propuestas en la declaración PRISMA (Urrútia y Bonfill, 2010) para la realización de revisiones sistemáticas. A continuación, se detallará el proceso llevado a cabo en sus distintas fases (ver Figura 1).

La búsqueda inicial se realizó el 22 de diciembre de 2018 combinando los términos prosocial ${ }^{*}$, empathy*, intervention y autism ${ }^{*}$ en la base de datos Web of Science (WOS) mediante el operador boleano AND. Lo poco encontrado contenía una gran limitación muestral y contextual, pues en la mayoría de los casos se centraban en población infantil, en un contexto escolar, y, además, estaban enfocadas en técnicas específicas. La búsqueda sistemática se realizó entre los meses de diciembre de 2018 y marzo de 2019 en las bases de datos WOS, PubMed, ScienceDirect y Dialnet, incluyendo como tipología documental artículos científicos publicados desde diciembre de 2018 hasta 1943 fecha en que se inició el estudio científico del autismo con el primer artículo científico obra Leo Kanner (Artigas-Pallares y Paula, 2012). Las combinaciones de términos utilizadas fueron las siguientes:

- prosocial $^{*}$ AND intervention AND autism ${ }^{*}$ y empathy* AND intervention AND autism* en el campo de tema para la WOS.

- prosocial $^{*}$ AND intervention AND autism ${ }^{*}$ y empathy* AND intervention AND autism* en los campos de título/abstract para PubMed.

- prosocial $^{*}$ AND intervention AND autism ${ }^{*}$ y empathy* AND intervention AND autism* en los campos de título, abstract y palabras clave con artículos de investigación para ScienceDirect.

- prosocial ${ }^{*} \mathrm{Y}$ intervención $\mathrm{Y}$ autismo* y empatía* $\mathrm{Y}$ intervención $\mathrm{Y}$ autismo* para Dialnet.

Como resultado se obtuvieron 272 artículos: 202 en la WOS, 43 en PubMed, 19 en ScienceDirect y 8 en Dialnet. Los trabajos fueron descargados en un archivo de texto y posteriormente fueron analizados en una hoja Excel, incluyendo las categorías de autores, título del trabajo, resumen y revista. Antes de empezar con la lectura de los 
resúmenes y la selección de artículos, se definieron los criterios de inclusión y de exclusión. A la revisión fueron incorporados artículos de investigaciones empíricas sobre intervenciones en prosocialidad y en empatía dirigidos a personas diagnosticadas con TEA, en cualquier idioma y publicados desde 1943 hasta 2018, ambos inclusive. Entre los criterios de exclusión, además de los derivados de los propios criterios de inclusión fueron excluidas otras tipologías documentales como actas de congresos, libros, editoriales, entre otros.

Tras un primer cribado donde se pasó a la lectura de los resúmenes de todos los artículos seleccionados y después de eliminar los duplicados, solo 56 fueron considerados aptos. Después de esto, se pasó a realizar un análisis del cuerpo de los artículos y con ello se eliminaron 22, principalmente por no incluir investigaciones empíricas de intervenciones en prosocialidad y empatía $(n=5)$, por no incluir personas diagnosticadas con TEA en la muestra $(n=8)$ y por problemas con el acceso al documento entero (n =9). Finalmente, los 34 artículos que cumplieron con los criterios de inclusión fueron seleccionados para llevar a cabo la revisión sistemática.

Todo este proceso de análisis para determinar la adecuación o no de los artículos al objetivo de la investigación se realizó de forma independiente por parte de dos investigadores de reconocido prestigio, que actuaron como evaluadores "ciegos". En los casos en los que existía desacuerdo acerca de la inclusión del trabajo en la revisión sistemática un tercer investigador participaba en la toma de decisión, siendo aceptado o no el trabajo por decisión de la mayoría de los evaluadores.

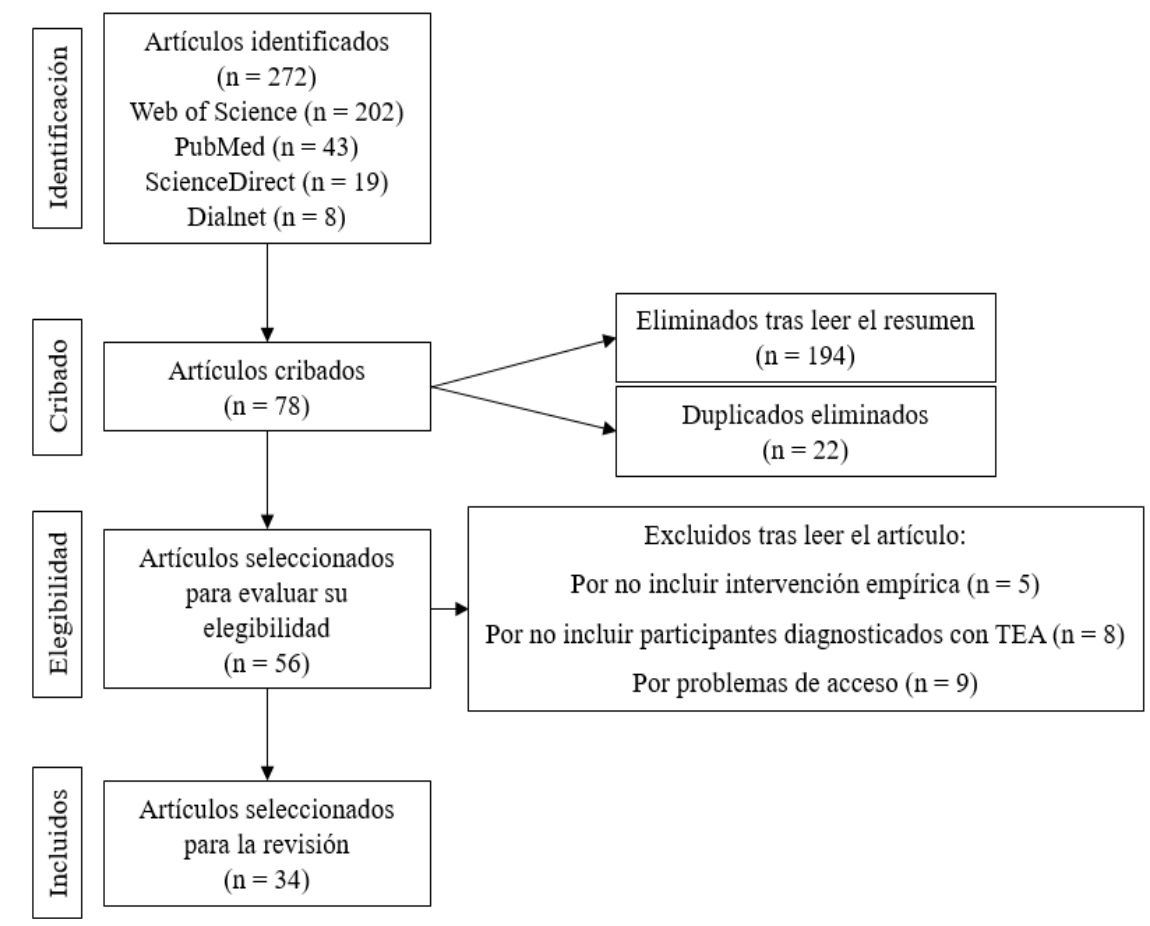

Figura 1. Diagrama de flujo según PRISMA. 


\section{Resultados}

La síntesis de los resultados de los estudios seleccionados se muestra en la Tabla 1, estructurada de forma cronológica y alfabética.

Tabla 1

Síntesis de los artículos revisados

\begin{tabular}{|c|c|c|c|c|}
\hline Autores y año & $\begin{array}{c}\text { Tipo de } \\
\text { intervención }\end{array}$ & $\begin{array}{c}\text { Frecuencia sesión/ } \\
\text { semana } \\
\text { Duración } \\
\text { Presencia grupo } \\
\text { control } \\
\end{array}$ & $\begin{array}{l}\text { Muestra: } \\
\text { sujetos con } \\
\text { TEA }\end{array}$ & Resultados \\
\hline $\begin{array}{l}\text { Anderson y } \\
\text { Meints (2016) }\end{array}$ & $\begin{array}{l}\text { Actividad asistida } \\
\text { con caballos }\end{array}$ & $\begin{array}{c}1 \\
5 \text { semanas } \\
\text { No }\end{array}$ & 15 (5-16 años) & $\begin{array}{c}\text { Incremento de la empatía } \\
\text { Disminución de los } \\
\text { comportamientos } \\
\text { adaptativos negativos } \\
\text { Mantenimiento } \\
\text { en habilidades de } \\
\text { comunicación y } \\
\text { socialización } \\
\end{array}$ \\
\hline $\begin{array}{c}\text { Andrews et al. } \\
\text { (2013) }\end{array}$ & $\begin{array}{l}\text { Intervención } \\
\text { cognitivo- } \\
\text { conductual }\end{array}$ & $\begin{array}{c}1 \\
5 \text { semanas } \\
\text { Sí }\end{array}$ & 58 (7-12 años) & $\begin{array}{l}\text { Mayor afecto apropiado } \\
\text { hacia otros Diferencias } \\
\text { significativas entre grupos } \\
\text { experimental y control }\end{array}$ \\
\hline $\begin{array}{l}\text { Argott et al. } \\
\text { (2007) }\end{array}$ & $\begin{array}{l}\text { Programa de } \\
\text { desvanecimiento } \\
\text { de guiones }\end{array}$ & $\begin{array}{c}3 \\
8 \text { semanas } \\
\text { No }\end{array}$ & 3 (11-14 años) & $\begin{array}{c}\text { Aumento de declaraciones } \\
\text { verbales de empatía }\end{array}$ \\
\hline $\begin{array}{l}\text { Baghdadli et } \\
\text { al. (2010) }\end{array}$ & $\begin{array}{l}\text { Intervenciones } \\
\text { prosociales }\end{array}$ & $\begin{array}{c}1 \\
20 \text { semanas } \\
\text { Sí }\end{array}$ & 13 (8-12 años) & $\begin{array}{c}\text { Mantenimiento } \\
\text { reconocimiento facial y } \\
\text { razonamiento social en } \\
\text { ambos grupos } \\
\text { No diferencias intergrupo }\end{array}$ \\
\hline $\begin{array}{l}\text { Becker et al. } \\
\quad(2017)\end{array}$ & $\begin{array}{l}\text { Terapia asistida } \\
\text { con animales }\end{array}$ & $\begin{array}{l}1 \\
12 \text { semanas } \\
\text { Sí }\end{array}$ & 31 (8-14 años) & $\begin{array}{c}\text { Menor presencia de } \\
\text { síntomas depresivos } \\
\text { Mayores habilidades de } \\
\text { teoría de la mente } \\
\text { Mantenimiento en } \\
\text { habilidades sociales }\end{array}$ \\
\hline $\begin{array}{l}\text { Begeer et al. } \\
\text { (2010) }\end{array}$ & $\begin{array}{l}\text { Entrenamiento en } \\
\text { teoría de la mente }\end{array}$ & $\begin{array}{c}1 \\
16 \text { semanas } \\
\text { Sí }\end{array}$ & 36 (8-13 años) & $\begin{array}{c}\text { Aumento en habilidades de } \\
\text { teoría de la mente } \\
\text { Mantenimiento del } \\
\text { comportamiento social y } \\
\text { habilidades empáticas }\end{array}$ \\
\hline Chen (2015) & $\begin{array}{c}\text { Vídeo -modelado } \\
\text { basado en realidad } \\
\text { aumentada }\end{array}$ & $\begin{array}{c}2 \\
\text { Un mes } \\
\text { No } \\
\end{array}$ & 6 (11-13 años) & $\begin{array}{c}\text { Mayor atención en relación } \\
\text { con las expresiones faciales } \\
\text { y las emociones }\end{array}$ \\
\hline $\begin{array}{l}\text { Chung et al. } \\
\text { (2016) }\end{array}$ & $\begin{array}{c}\text { Juego prosocial en } \\
\text { línea }\end{array}$ & $\begin{array}{l}3 \\
6 \text { semanas } \\
\text { Sí }\end{array}$ & 20 (13-18 años) & $\begin{array}{c}\text { Mayor comunicación } \\
\text { social, respuesta correcta } \\
\text { de palabras emocionales y } \\
\text { emociones faciales }\end{array}$ \\
\hline
\end{tabular}




\begin{tabular}{|c|c|c|c|c|}
\hline Autores y año & $\begin{array}{c}\text { Tipo de } \\
\text { intervención }\end{array}$ & $\begin{array}{l}\text { Frecuencia sesión/ } \\
\text { semana } \\
\text { Duración } \\
\text { Presencia grupo } \\
\text { control } \\
\end{array}$ & $\begin{array}{l}\text { Muestra: } \\
\text { sujetos con } \\
\text { TEA }\end{array}$ & Resultados \\
\hline $\begin{array}{l}\text { Cook et al. } \\
\text { (2018) }\end{array}$ & $\begin{array}{c}\text { Contacto musical } \\
\text { en la escuela }\end{array}$ & $\begin{array}{c}1 \\
11 \text { semanas } \\
\text { No }\end{array}$ & $\begin{array}{l}49 \text { normotípicos } \\
\text { y } 10 \text { con TEA } \\
\text { (10-11 años })\end{array}$ & $\begin{array}{l}\text { Aumento en prosocialidad, } \\
\text { simpatía, tendencia a abusar } \\
\text { Se mantiene la tendencia a } \\
\text { ser una víctima }\end{array}$ \\
\hline $\begin{array}{c}\text { Crozier y } \\
\text { Tincani (2006) }\end{array}$ & Historias sociales & $\begin{array}{c}3 \\
3 \text { semanas } \\
\text { No }\end{array}$ & 3 (3-5 años) & $\begin{array}{c}\text { Disminución del } \\
\text { comportamiento social } \\
\text { inadecuado } \\
\text { Aumento del } \\
\text { comportamiento social } \\
\text { adecuado } \\
\end{array}$ \\
\hline $\begin{array}{l}\text { Di Renzo et al. } \\
\text { (2015) }\end{array}$ & $\begin{array}{l}\text { Programa a largo } \\
\text { plazo basado en la } \\
\text { relación }\end{array}$ & $\begin{array}{c}10 \text { horas/semana } \\
4 \text { años } \\
\text { No }\end{array}$ & 90 (2-16 años) & $\begin{array}{l}\text { Mayor cociente intelectual, } \\
\text { razonamiento fluido } \\
\text { Disminución de los síntomas } \\
\text { del TEA }\end{array}$ \\
\hline $\begin{array}{l}\text { Gantman et al. } \\
\qquad(2011)\end{array}$ & $\begin{array}{l}\text { Programa de } \\
\text { habilidades } \\
\text { sociales PEERS }\end{array}$ & $\begin{array}{l}1 \\
14 \text { semanas } \\
\text { Sí }\end{array}$ & 17 (18-23 años) & $\begin{array}{c}\text { Mayores habilidades } \\
\text { sociales } \\
\text { Disminución del sentimiento } \\
\text { de soledad } \\
\text { Grupo experimental obtuvo } \\
\text { mejores resultados }\end{array}$ \\
\hline $\begin{array}{l}\text { Goldingay et } \\
\text { al. (2013) }\end{array}$ & $\begin{array}{l}\text { Juego de } \\
\text { simulación }\end{array}$ & $\begin{array}{c}1 \\
8 \text { semanas } \\
\text { No }\end{array}$ & $\begin{array}{c}5 \text { (media de } 13 \\
\text { años) }\end{array}$ & $\begin{array}{c}\text { Mayor autorregulación, } \\
\text { empatía } \\
\text { Disminución de la } \\
\text { hiperactividad } \\
\text { Mantenimiento en } \\
\text { pensamiento } \\
\text { flexible } \\
\end{array}$ \\
\hline $\begin{array}{l}\text { Guivarch et al. } \\
\text { (2017) }\end{array}$ & $\begin{array}{l}\text { Entrenamiento } \\
\text { de habilidades } \\
\text { sociales implícitas }\end{array}$ & $\begin{array}{c}1 \\
22 \text { semanas } \\
\text { No }\end{array}$ & 6 (9-10 años) & $\begin{array}{c}\text { Mayor adaptación general y } \\
\text { habilidades sociales } \\
\text { Disminución de los síntomas } \\
\text { del TEA }\end{array}$ \\
\hline $\begin{array}{l}\text { Halle et al. } \\
\quad(2016)\end{array}$ & $\begin{array}{l}\text { Modelado en } \\
\text { vídeo de historias } \\
\text { sociales }\end{array}$ & $\begin{array}{c}2 \\
6 \text { semanas } \\
\text { No }\end{array}$ & 4 (8 años) & $\begin{array}{c}\text { Incremento en la frecuencia } \\
\text { de respuesta de saludo } \\
\text { apropiadas } \\
\text { Generalización a otros } \\
\text { contextos }\end{array}$ \\
\hline $\begin{array}{l}\text { Henning et al. } \\
\qquad(2016)\end{array}$ & $\begin{array}{l}\text { Intervención } \\
\text { basada en el juego }\end{array}$ & $\begin{array}{c}9 \text { semanas } \\
\text { No }\end{array}$ & $\begin{array}{l}5 \text { TEA y } 5 \\
\text { normotípicos } \\
(4-11 \text { años })\end{array}$ & Resultados contradictorios \\
\hline $\begin{array}{l}\text { Kamps et al. } \\
\qquad(2014)\end{array}$ & $\begin{array}{l}\text { Mediación entre } \\
\text { compañeros e } \\
\text { instrucción directa }\end{array}$ & $\begin{array}{l}2 \\
6 \text { meses } \\
\text { Sí }\end{array}$ & 95 (5-6 años) & $\begin{array}{c}\text { Aumento del lenguaje, } \\
\text { comunicación adaptativa, } \\
\text { prosocialidad }\end{array}$ \\
\hline $\begin{array}{l}\text { Koch et al. } \\
\text { (2014) }\end{array}$ & $\begin{array}{l}\text { Terapia de } \\
\text { movimientos de } \\
\text { baile }\end{array}$ & $\begin{array}{c}1 \\
7 \text { semanas } \\
\text { Sí }\end{array}$ & 31 (16-47 años) & $\begin{array}{c}\text { Aumento en bienestar, } \\
\text { conciencia corporal, } \\
\text { conciencia del otro, } \\
\text { habilidades sociales } \\
\text { Mantenimiento en empatía } \\
\text { Mejores resultados en grupo } \\
\text { experimental }\end{array}$ \\
\hline
\end{tabular}




\begin{tabular}{|c|c|c|c|c|}
\hline Autores y año & $\begin{array}{c}\text { Tipo de } \\
\text { intervención }\end{array}$ & $\begin{array}{c}\text { Frecuencia sesión/ } \\
\text { semana } \\
\text { Duración } \\
\text { Presencia grupo } \\
\text { control } \\
\end{array}$ & $\begin{array}{l}\text { Muestra: } \\
\text { sujetos con } \\
\text { TEA }\end{array}$ & Resultados \\
\hline $\begin{array}{l}\text { Koegel et al. } \\
\text { (2015) }\end{array}$ & Vídeo-feedback & $\begin{array}{l}1 \\
5,9 \text { y } 6 \text { semanas } \\
\text { No }\end{array}$ & 3 (19-26 años) & $\begin{array}{c}\text { Mayor expresión verbal, } \\
\text { frecuencia de preguntas } \\
\text { empáticas, empatía, } \\
\text { seguridad en las habilidades } \\
\text { comunicativas }\end{array}$ \\
\hline $\begin{array}{l}\text { Koehne et al. } \\
\text { (2015) }\end{array}$ & $\begin{array}{c}\text { Imitación y } \\
\text { sincronización } \\
\text { basado en la danza } \\
\text { y el movimiento } \\
\text { (SI-DMI) }\end{array}$ & $\begin{array}{l}1 \\
10 \text { semanas } \\
\text { Sí }\end{array}$ & 55 (18-55 años) & $\begin{array}{l}\text { Mayor inferencia de } \\
\text { emociones } \\
\text { Mantenimiento de los } \\
\text { sentimientos de empatía } \\
\text { Mejores resultados en grupo } \\
\text { SI-DMI en comparación con } \\
\text { grupo CMI (intervención de } \\
\text { control de movimiento) }\end{array}$ \\
\hline $\begin{array}{l}\text { Kroeger et al. } \\
\quad(2006)\end{array}$ & $\begin{array}{l}\text { Enseñanza } \\
\text { directa en juegos } \\
\text { vs. juegos no } \\
\text { estructurados } \\
\end{array}$ & $\begin{array}{c}3 \\
5 \text { semanas } \\
\text { Sí }\end{array}$ & 25 (4-6 años) & $\begin{array}{l}\text { Aumento en prosocialidad } \\
\text { Grupo de enseñanza directa } \\
\text { logró mayores avances }\end{array}$ \\
\hline $\begin{array}{l}\text { LaCava et al. } \\
\quad(2010)\end{array}$ & $\begin{array}{c}\text { Programa } \\
\text { informático Mind } \\
\text { Reading }\end{array}$ & $\begin{array}{l}1 \\
10 \text { semanas } \\
\text { No }\end{array}$ & 4 (7-10 años) & $\begin{array}{l}\text { Incremento en interacciones } \\
\text { sociales positivas }\end{array}$ \\
\hline $\begin{array}{l}\text { Mastrominico } \\
\text { et al. (2018) }\end{array}$ & $\begin{array}{l}\text { Movimientos de } \\
\text { danza reflejados } \\
\text { en el espejo }\end{array}$ & $\begin{array}{l}1 \\
10 \text { semanas } \\
\text { Sí }\end{array}$ & 56 (14-52 años) & $\begin{array}{c}\text { No diferencias significativas } \\
\text { entre grupos }\end{array}$ \\
\hline $\begin{array}{l}\text { McVey et al. } \\
\quad(2016)\end{array}$ & $\begin{array}{l}\text { Programa de } \\
\text { habilidades } \\
\text { sociales } \\
\text { PEERS }\end{array}$ & $\begin{array}{l}1 \\
16 \text { semanas } \\
\text { Sí }\end{array}$ & 56 (18-28 años) & $\begin{array}{c}\text { Aumento de la capacidad } \\
\text { de respuesta social, empatía, } \\
\text { interacciones directas } \\
\text { Disminución de la ansiedad } \\
\text { social } \\
\end{array}$ \\
\hline $\begin{array}{l}\text { O'Haire et al. } \\
\quad(2013)\end{array}$ & $\begin{array}{l}\text { Actividad asistida } \\
\text { con animales }\end{array}$ & $\begin{array}{l}2 \\
1 \text { año } \\
\text { Sí }\end{array}$ & $\begin{array}{l}33 \text { con TEA y } 66 \\
\text { normotípicos } \\
\text { (5-12 años) }\end{array}$ & $\begin{array}{c}\text { Mayor prosocialidad y } \\
\text { afecto positivo } \\
\text { Disminución de } \\
\text { comportamientos centrados } \\
\text { en sí mismos, afectos } \\
\text { negativos } \\
\end{array}$ \\
\hline $\begin{array}{l}\text { Rabin et al. } \\
\qquad(2018)\end{array}$ & $\begin{array}{l}\text { Programa de } \\
\text { habilidades } \\
\text { sociales PEERS }\end{array}$ & $\begin{array}{l}1 \\
14 \text { semanas } \\
\text { Sí }\end{array}$ & 41 (12-17 años) & $\begin{array}{c}\text { Aumento en empatía y } \\
\text { habilidades sociales } \\
\text { Disminución síntomas del } \\
\text { TEA } \\
\end{array}$ \\
\hline $\begin{array}{l}\text { Rosenbloom et } \\
\text { al. (2016) }\end{array}$ & $\begin{array}{l}\text { Aplicación } \\
\text { I-Connect para el } \\
\text { autocontrol }\end{array}$ & $\begin{array}{l}2-3 \\
9 \text { semanas } \\
\text { No }\end{array}$ & 1 (9 años) & $\begin{array}{c}\text { Aumento del tiempo de } \\
\text { tarea } \\
\text { Disminución de } \\
\text { la frecuencia de } \\
\text { comportamientos } \\
\text { desafiantes }\end{array}$ \\
\hline $\begin{array}{l}\text { Soorya et al. } \\
\text { (2015) }\end{array}$ & $\begin{array}{l}\text { Entrenamiento } \\
\text { en habilidades } \\
\text { sociales }\end{array}$ & $\begin{array}{l}1 \\
12 \text { semanas } \\
\text { Sí }\end{array}$ & 69 (8-11 años) & $\begin{array}{c}\text { Mayor conducta social, } \\
\text { comunicación no verbal, } \\
\text { empatía, relaciones sociales } \\
\text { Mantenimiento de la } \\
\text { cognición social }\end{array}$ \\
\hline
\end{tabular}




\begin{tabular}{|c|c|c|c|c|}
\hline Autores y año & $\begin{array}{c}\text { Tipo de } \\
\text { intervención }\end{array}$ & $\begin{array}{c}\text { Frecuencia sesión/ } \\
\text { semana } \\
\text { Duración } \\
\text { Presencia grupo } \\
\text { control }\end{array}$ & $\begin{array}{l}\text { Muestra: } \\
\text { sujetos con } \\
\text { TEA }\end{array}$ & Resultados \\
\hline $\begin{array}{l}\text { Sung et al. } \\
\text { (2018) }\end{array}$ & $\begin{array}{l}\text { Programa de } \\
\text { habilidades } \\
\text { sociales }\end{array}$ & $\begin{array}{c}1 \\
8 \text { semanas } \\
\text { No }\end{array}$ & 17 (18-25 años) & $\begin{array}{c}\text { Aumento en conocimiento } \\
\text { de habilidades sociales, } \\
\text { funcionamiento social y } \\
\text { autoeficacia social/empática }\end{array}$ \\
\hline $\begin{array}{l}\text { Tsui y } \\
\text { Rutherford } \\
\text { (2014) }\end{array}$ & $\begin{array}{l}\text { Automodelado en } \\
\text { vídeo }\end{array}$ & $\begin{array}{l}3 \\
4 \text { semanas } \\
\text { No }\end{array}$ & 1 (30 años) & $\begin{array}{c}\text { Disminución de la tendencia } \\
\text { a invadir el espacio } \\
\text { personal, temblores al } \\
\text { interactuar con otros } \\
\text { Aumento de saludos } \\
\text { verbales, contacto visual }\end{array}$ \\
\hline $\begin{array}{l}\text { Tsunemi et al. } \\
\qquad(2014)\end{array}$ & $\begin{array}{c}\text { Narrativa en libros } \\
\text { de cuentos }\end{array}$ & $\begin{array}{c}6 \\
1 \text { semana } \\
\text { Sí } \\
\end{array}$ & 16 (9 años) & $\begin{array}{c}\text { Aumento en la capacidad de } \\
\text { toma de roles }\end{array}$ \\
\hline $\begin{array}{l}\text { Vesperini et al. } \\
\text { (2014) }\end{array}$ & $\begin{array}{l}\text { Análisis de } \\
\text { secuencias de } \\
\text { vídeo }\end{array}$ & $\begin{array}{c}20 \text { sesiones en } 6 \\
\text { meses } \\
\text { No }\end{array}$ & 16 (8-15 años) & $\begin{array}{l}\text { Incremento en habilidades } \\
\text { de comunicación en } \\
\text { grupo, hogar y escuela, } \\
\text { comportamiento } \\
\text { socioadaptativo, autonomía } \\
\text { social, actividades de ocio }\end{array}$ \\
\hline $\begin{array}{l}\text { Yoo y Kim } \\
\quad(2018)\end{array}$ & $\begin{array}{l}\text { Intervención } \\
\text { mediada por el } \\
\text { ritmo }\end{array}$ & $\begin{array}{c}1 \\
8 \text { semanas } \\
\text { No }\end{array}$ & $\begin{array}{l}8 \text { (10 años de } \\
\text { media) }\end{array}$ & $\begin{array}{c}\text { Incremento en cooperación } \\
\text { y autocontrol } \\
\text { Disminuciónde la asincronía } \\
\text { Mantenimiento en } \\
\text { asertividad }\end{array}$ \\
\hline $\begin{array}{l}\text { Zlomke et al. } \\
\text { (2017) }\end{array}$ & $\begin{array}{l}\text { PCIT (Parent-Child } \\
\text { Interaction Therapy }\end{array}$ & $\begin{array}{c}1 \\
19 \text { semanas } \\
\text { No }\end{array}$ & 17 (2-8 años) & $\begin{array}{c}\text { Disminución síntomas del } \\
\text { TEA } \\
\text { Mayores habilidades } \\
\text { sociales } \\
\text { No diferencias en } \\
\text { comunicación funcional }\end{array}$ \\
\hline
\end{tabular}

La mayoría de los estudios señalan que es posible un incremento en el área prosocial y empática en las personas con TEA mediante diferentes técnicas, que sirven de nexo para la presentación y adecuada compresión de los resultados.

Tres de los artículos emplearon técnicas de modelado en vídeo en sus intervenciones, con una posterior interpretación conjunta de las secuencias entre sujetos y terapeutas (Vesperini et al., 2014; Chen, Lee y Lin, 2015; Halle, Ninnes, Ninnes y Lawson, 2016). Los resultados fueron positivos en todos los casos, produciéndose incluso generalización a otros contextos. Asimismo, otros dos de los artículos emplean este mismo soporte mediante automodelado (Tsui y Rotherford, 2014; Koegel, Aasbaugh, Navab y Koegel, 2015) en las que se muestra a los sujetos realizando conductas prosociales (como mantener contacto ocular, devolver un saludo, mantener la distancia adecuada o expresiones verbales empáticas) entrenadas previamente. Los resultados mostraron mejoras en todas las áreas trabajadas mediante esta técnica.

Tres de los artículos analizados trabajan la prosocialidad mediante terapia asistida con animales (O'Haire, McKenzie, Beck y Slaughter, 2013; Anderson y Meints, 2016; 
Becker, Rogers y Burrows, 2017). Los resultados obtenidos en el primer y tercer caso fueron positivos para los grupos experimentales en comparación con el grupo control en la mayoría de las áreas prosociales trabajadas; en el segundo artículo, no obstante, las mejoras fueron limitadas.

Cinco artículos emplearon diferentes técnicas artísticas en sus intervenciones (Koch, Mehl, Sobanski, Sieber y Fuchs, 2014; Koehne, Behrends, Fairhurst y Dziobek, 2015; Mastrominico et al., 2018; Cook, Ogden y Winstone, 2018; Yoo y Kim, 2018). Estas técnicas incluían terapia de danza basada en el movimiento reflejado en el espejo para el primer y tercer artículo, e imitación y sincronización basados en la danza y el movimiento, terapia basada en el contacto musical y terapia mediada por el ritmo respectivamente para los restantes. En ningún caso se obtuvieron resultados positivos para todas las áreas trabajadas, aunque en el caso de aquellos artículos que contaban con la presencia de grupo control, se pudieron apreciar diferencias significativas positivas para los grupos experimentales (excepto en el caso de Mastrominico et al., (2018), que no existieron diferencias). Además, en la intervención de Cook et al. (2018), pudieron apreciarse mejora en los niños normotípicos que tuvieron contacto con los niños con TEA.

Cuatro de los artículos se valían de soportes informáticos para la puesta en marcha de sus intervenciones (LaCava, Rankin, Mahlios, Cook y Simpson, 2010; Goldingay et al., 2013; Chung, Han, Shin y Renshaw, 2016; Rosenbloom, Mason, Wills y Mason, 2016). Estos grupos emplearon una guía interactiva informática denominada Mind Reading, un juego de simulación, un juego en línea y una aplicación de autocontrol de entrega de mensajes personalizables respectivamente. Los resultados fueron favorables para todos los estudios en prácticamente todas las áreas trabajadas, y en el caso de Chung et al. (2016), no se apreciaron diferencias entre el grupo experimental y el grupo control (que empleaba el mismo juego fuera de línea).

Siete de los artículos utilizaron programas de habilidades sociales como método de intervención (Baghdadli, Brisot-Dubois, Picot y Michelon, 2010; Gantman, Kapp, Orenski y Laugeson, 2011; Soorya et al., 2015; McVey et al., 2016; Guivarch et al., 2017; Rabin, Israel-Yaacov, Laugeson, Mor-Snir y Golan, 2018; Sung et al., 2018). Gantman et al. (2011, McVey et al. (2016) y Rabin et al. (2018) emplearon un programa conocido como PEERS for Young Adults, mostrando resultados favorables en todas las áreas trabajadas en las tres intervenciones. En cuanto al resto, utilizaron diversos programas, que en el primero de los casos no produjo diferencias entre grupo experimental y control y las mejoras fueron limitadas. En los artículos restantes, los resultados fueron positivos en la mayoría de las áreas trabajadas.

Dos artículos se valieron de intervenciones mediante exposiciones a historias sociales (Tsunemi, Tamura, Ogawa, Isomura y Masataka, 2014; Crozier y Tincani, 2016). En ambos casos los resultados fueron favorables en las áreas trabajadas y, además, se apreciaron diferencias significativas positivas entre grupo experimental y grupo control para el primero de ellos.

Dos artículos emplearon técnicas de instrucción directa como lecciones programadas de habilidades de comunicación específicas y pautas de texto escritas (Kroeger et al., 2006; Kamps et al., 2014). Los resultados fueron positivos en todas las áreas trabajadas 
y, además, el grupo experimental del grupo de Kroeger et al. (2006) obtuvo mejores calificaciones académicas en comparación con el grupo control.

El resto de los artículos empleaban técnicas diversas. Argott, Townsend, Sturney y Poulson (2007) implementaron un programa de desvanecimiento de guiones para mejorar la respuesta empática, obteniendo resultados favorables. Beeger et al. (2010) realizaron una intervención mediante un entrenamiento en teoría de la mente, cuyos resultados fueron positivos únicamente en esta área para el grupo experimental en comparación con el control. Andrews, Attwood y Sofronoff (2013) llevaron a cabo una intervención-cognitivo conductual que se tradujo en resultados satisfactorios y, en concreto, para el grupo experimental. Di Renzo, Di Castelbianco, Petrillo, Racinaro y Rea (2015) llevaron a cabo un programa de cuatro años que incluía trabajo en casa, terapia con animales, asesoramiento para los padres, apoyo en la escuela, terapia ambulatoria que comprendía sesiones individuales y grupales, musicoterapia, psicomotricidad, psicoterapia y trabajo pedagógico entre otros. Los resultados mostraron una mejora en el cociente intelectual, razonamiento fluido y síntomas del TEA en toda la muestra. Henning, Cordier, Wilkes-Gillan y Falkmer (2016) llevaron a cabo una intervención basada en el juego. Los resultados indican contradicción, pues la terapia fue efectiva para 2 niños con TEA y 3 de sus compañeros de juego, tuvo un efecto negativo en 3 de los niños con TEA, y ningún efecto observable en los otros 2 compañeros de juego. Por último, Zlomke, Jeter y Murphy (2017) llevaron a cabo una intervención mediante una terapia de interacción padres-hijos denominada PICT (Parent-Child Interaction Therapy) basada en el comportamiento y que se enfocaba en la relación entre padres e hijos a través del entrenamiento en vivo de las interacciones del juego y la implementación de técnicas de disciplina consistentes, aunque los resultados fueron positivos únicamente en dos de las tres áreas trabajadas.

\section{Discusión y conclusiones}

El objetivo de la esta revisión es determinar la efectividad de las diferentes intervenciones dirigidas a mejorar la prosocialidad y la empatía en personas con TEA, así como identificar posibles factores determinantes. Para ello se va a llevar a cabo un análisis de las mismas, así como una valoración de sus puntos fuertes y de sus limitaciones, a partir las diferentes técnicas y programas empleados.

En cuanto al modelado en vídeo empleado por algunos de los autores (Tsui y Rotherford, 2014; Vesperini et al., 2014; Chen et al., 2015; Koegel et al., 2015; Halle et al., 2016), Corbett (2003) afirma que el modelado en vídeo presenta una mayor efectividad en adquisición, mantenimiento y generalización en individuos con TEA que el modelado en vivo. Estos resultados positivos pueden apreciarse en el primer, tercero y cuarto estudio.

En relación con la terapia asistida con animales, empleada por (O'Haire, McKenzie, Beck y Slaughter, 2013; Anderson y Meints, 2016; Becker, Rogers y Burrows, 2017), Jenkins y Reed (2013) postulan como uno de los factores clave en este tipo de terapias el contenido de las sesiones. Becker et al. (2017) afirman que existen estudios que demuestran que el aumento producido en la prosocialidad es debido a cambios 
fisiológicos favorables tales como la disminución de la frecuencia cardiaca y de los niveles de cortisol.

Por otra parte, de los artículos que empleaban técnicas artísticas en sus intervenciones, los grupos de Koch et al. (2014) y de Mastrominico et al. (2018) emplean una técnica de movimientos de danza basados en el reflejo del espejo. McGarry y Russo (2011) afirman que el aumento de la empatía mediante este método podría estar mediado por un sistema de retroalimentación de movimientos emocionales que involucra circuitos de neuronas espejo. Así, el resultado sería una mayor comprensión de las intenciones ajenas al sentir el propio sujeto estas mismas emociones. Sin embargo, ninguno de los dos trabajos obtuvo mejoras significativas. En cuanto al grupo de Koehne et al. (2015), la técnica utilizada fue similar y, de nuevo, la empatía tampoco aumentó, aunque sí la inferencia de emociones. El grupo de Cook et al. (2018) contaba con estudios previos (Gerry, Unrau y Trainor, 2012; Rabinowitch, Cross y Burnard, 2013) que se basaban en el contacto musical y que obtuvieron grandes beneficios. Cook et al. (2018) también obtuvo resultados positivos en empatía, pero contaba con la limitación de la ausencia de un grupo control. Lo mismo sucedió en la intervención mediada por el ritmo de Yoo y Kim (2018), donde el beneficio se reflejó en un aumento de la cooperación y del autocontrol, aunque con las limitaciones del pequeño tamaño de la muestra y de la ausencia del grupo control.

En cuanto al uso de soportes informáticos, Lozano y Alcaraz (2011) afirman que existe un amplio cuerpo de estudios que respaldan el uso de entornos informáticos para el incremento de las competencias emocionales en personas con TEA, ya que aportan una visión estructurada de la realidad y les permite el control del entorno y su participación en contingencias perfectas y comprensibles. Así, los grupos de LaCava et al. (2010), Goldingay et al. (2013) y Chung et al. (2016) encontraron efectos positivos. Rosenbloom et al. (2016), también utilizaron una plataforma multimedia mediante el uso de la aplicación de autocontrol I-Conncect. Para ello, se basaron en estudios como los de Crutchfield, Mason, Chambers, Wills y Mason (2015) que obtuvieron resultados positivos mediante el uso de esta aplicación.

Los programas de habilidades sociales como métodos de intervención, como es el caso de Baghdadli et al. (2010), Gantman et al. (2011), Soorya et al. (2015), McVey et al. (2016), Guivarch et al. (2017), Rabin et al. (2018) y Sung et al. (2018), exceptuando el primero, obtuvieron resultados positivos en prácticamente todas las áreas trabajadas.

En cuanto al uso de exposición a historias sociales, basándose en el estudio de McKenzie, Evans y Handley (2011), que hipotetizaban con la idea de que las personas con TEA tienen una capacidad menor para integrar el conocimiento contextual relevante y disponible en el proceso de razonamiento, los hallazgos encontrados en el estudio de Tsunemi et al. (2014) proporcionan evidencias que respaldan esta hipótesis. Es decir, el ayudar a estos niños a comprender las actividades de los personajes de las historias les brinda la oportunidad de entender los estados mentales de los demás. También Crozier y Tincani (2006) utilizaron las historias sociales con resultados positivos en niños con TEA.

Argott et al. (2007) emplearon un procedimiento de desvanecimiento de guiones para enseñar habilidades de respuesta empática, basándose en estudios previos como el de Stevenson, Krantz y McClannahan (2000), en el que se introducían guiones en 
audio que luego se iban desvaneciendo progresivamente para enseñar a un grupo de niños con TEA a mantener conversaciones con un adulto. Los resultados en este caso también fueron positivos, pues los efectos se observaron en interacciones sin guion y se mantuvieron en el tiempo. Sin embargo, el grupo de Argott et al. (2007) señala limitaciones como que estos procedimientos podían estar limitados únicamente a personas a las que previamente se les han enseñado estas habilidades, o que estas respuestas empáticas estarían centradas únicamente en tres categorías de afecto (felicidad, dolor y cansancio).

Begeer et al. (2010) utilizaron un entrenamiento en teoría de la mente con resultados positivos únicamente en esta área, aunque estudios previos como el de Gevers, Clifford, Mager y Boer (2006) habían obteniendo beneficios en percepción/imitación, simulación y comprensión del humor, comportamiento adaptativo y habilidades sociales.

Cooley, Veldorale-Griffin, Petren y Mullis (2014) llevaron a cabo un metaanálisis para estudiar la efectividad del PCIT (Parent-Child Interaction Therapy), el programa empleado por el equipo de Zlomke et al. (2017), y los resultados obtenidos demostraron su efectividad a la hora de reducir el comportamiento disruptivo en niños con desarrollo normotípico, aunque con ciertas diferencias individuales que hacían necesaria la investigación de este programa en poblaciones específicas. Los datos recogidos de la muestra de niños con TEA de Zlomke et al. (2017) también fueron positivos, aunque no para todas las áreas trabajadas.

Basándose en el estudio de McKenzie, Evans y Handley (2011), que hipotetizaban con la idea de que las personas con TEA tienen una capacidad menor para integrar el conocimiento contextual relevante y disponible en el proceso de razonamiento, los hallazgos encontrados en el estudio de Tsunemi et al. (2014) proporcionan evidencias que respaldan esta hipótesis. Es decir, el ayudar a estos niños a comprender las actividades de los personajes de las historias les brinda la oportunidad de entender los estados mentales de los demás. También Crozier y Tincani (2006) utilizaron las historias sociales con resultados positivos en niños con TEA. Cooley, Veldorale-Griffin, Petren y Mullis (2014) llevaron a cabo un metaanálisis para estudiar la efectividad del PCIT (Parent-Child Interaction Therapy), el programa empleado por el equipo de Zlomke et al. (2017), y los resultados obtenidos demostraron su efectividad a la hora de reducir el comportamiento disruptivo en niños con desarrollo normotípico, aunque con ciertas diferencias individuales que hacían necesaria la investigación de este programa en poblaciones específicas. Los datos recogidos de la muestra de niños con TEA de Zlomke et al. (2017) también fueron positivos, aunque no para todas las áreas trabajadas.

Estudios como el de Di Renzo et al. (2015) ponen de manifiesto la efectividad de las intervenciones a largo plazo y mediante un equipo. Este enfoque se postula como uno de los factores clave en la efectividad de los servicios de atención a niños con TEA. Por otra parte, varios estudios emplearon como muestra pacientes con TEA de alto funcionamiento, como el de Kamps et al. (2014), Vesperini et al. (2014) y Andrews et al. (2013) obteniéndose resultados positivos. Algunos autores atribuyen la efectividad en las intervenciones con personas con TEA de alto funcionamiento a un mayor CI y a una menor sintomatología del TEA (Kjellmer, Hedvall, Fernell, Gillberg \& Norrelgen, 2011). 
A la vista de los resultados se puede observar que en 31 de los artículos en los que se ha aplicado un programa para mejorar la prosocialidad y la empatía se producen mejoras. Sólo en dos trabajos no se producen diferencias (Mastrominico et al., 2008; Baghdadlii et al., 2010), y en uno de ellos los resultados son contradictorios (Henning et al. 2016).

Sin dudar acerca de la importancia de trabajar estas habilidades en sujetos con TEA, cabe tomar con precaución los resultados obtenidos por razones metodológicas principalmente. Con respecto a la intensidad del tratamiento sólo 12 trabajos incluyen dos o más sesiones de trabajo por semana, y son también 12 los que han aplicado una intervención mayor de doce o más semanas. Frecuencia muy por debajo a lo recomendado con sujetos TEA, la cual es de 20 horas de terapia a la semana (Weinmann et al., 2009). Así, la intervención que más se acerca a esta frecuencia mínima recomendada es la llevada a cabo por Di Renzo et al. (2015), que cuenta con unas 10 horas semanales. En 16 trabajos se ha empleado un grupo control y en 14 la muestra estaba formada por 20 o más sujetos. En 4 trabajos la muestra presenta una diferencia de más de 10 años entre los sujetos que participaron en el estudio.

Gresham, Sugai y Horner (2001) concluyen que para que una intervención sea efectiva debe cumplir los siguientes requisitos: 1) mayor frecuencia e intensidad en la implementación, 2) generalización y mantenimiento mediante su aplicación en entornos menos restringidos y descontextualizados, 3) individualización y adaptación de las intervenciones a las necesidades individuales y 4) fidelidad a la hora de efectuar las intervenciones para concluir su eficacia.

Atendiendo a estos criterios metodológicos solo tres trabajos los cumplirían: los llevados a cabo por O'Haire et al. (2013) empleando una actividad asistida con animales, el de Kamps et al. (2014) de mediación entre compañeros e instrucción directa y el de Di Renzo et al. (2015) que aplica un programa a largo plazo basado en la relación. Algunas de estas limitaciones metodológicas son recogidas por los autores, además de otras como la no generalización a otros contextos o, como apunta Corbett (2003) en el caso del modelado en vídeo en este tipo de colectivos, los comportamientos pueden ser retenidos mediante la memoria y empleados con total carencia de espontaneidad y flexibilidad en situaciones sociales complejas. Koch et al. (2014) señalan como limitaciones una muestra demasiado pequeña, la falta de aleatorización, un posible efecto de Hawthorne (mejora en el grupo de intervención debida a una mayor atención) y la falta de método de doble ciego. Begeer et al. (2010) señalan como posibles limitaciones los efectos de la edad, el CI o la severidad del trastorno.

Tras la integración de los resultados, se puede afirmar que existen multitud de técnicas efectivas para mejorar las conductas prosociales y empáticas en las personas con TEA. Los resultados fueron positivos en la mayoría de los estudios, pero se hace imposible una comparación de la efectividad de cada una de las intervenciones debido a las diferencias metodológicas existentes entre ellas, principalmente derivadas de la muestra, tamaño y características, y del número de sesiones de intervención. Sin embargo, parece que las variables responsables de la efectividad en las intervenciones no es tanto el tipo de terapia empleado, sino más bien el grado de lenguaje receptivo y expresivo (relacionado con el nivel cognitivo), las habilidades de comunicación no verbal (relacionadas con la severidad de los síntomas del TEA y el funcionamiento 
adaptativo), el razonamiento fluido (relacionado con los síntomas del TEA) y el CI, algo que se manifiesta en una mayor efectividad de las intervenciones con muestras compuestas por personas con TEA de alto funcionamiento.

Esta revisión deja entrever, en el caso del TEA, las diferencias metodológicas existentes entre unos trabajos y otros con respecto a la aplicación de programas de intervención. Muchos de estos trabajos adolecen de la falta de un grupo control y doble ciego, existen diferencias con respecto a las características de la muestra de los grupos TEA, como por ejemplo la edad de los participantes, lo que dificulta la generalización de los resultados. En todos ellos se puede observar, acorde a la propuesta de Smith et al. (2007), que todos los trabajos analizados se encontrarían en la fase de la evaluación inicial del programa, si bien cabe destacar la aplicación del programa PEERS en tres estudios, el cual puede ser un claro candidato sobre el cual aplicar las siguientes fases recogidas en dicho protocolo, si se tiene en cuenta las recomendaciones metodológicas de Chambless et al. (1996). En este sentido, y dado los resultados prometedores encontrados con respecto a mejoras en la prosocialidad y en las habilidades empáticas sería interesante continuar con dichos estudios evaluando la efectividad y eficacia a largo plazo, en diferentes contextos y en contextos comunitarios, controlando variables tan importantes en el caso del TEA como el CI, el sexo, la tenencia o no de lenguaje o el nivel de afectación del trastorno.

También cabe mencionar que este trabajo no queda exento de limitaciones, que en futuras investigaciones podrían solventarse mediante la utilización de un repertorio de palabras más amplio que recogiera una mayor cantidad de artículos a analizar, así como de más bases de datos que en la presente revisión. A su vez, sería relevante poder analizar las características particulares de cada programa de intervención en cuanto a metodología, objetivos, actividades, con vistas a conocer qué papel juegan en cuanto a los cambios en prosocialidad y empatía en sujetos TEA.

\section{Referencias}

Anderson, S. \& Meints, K. (2016). Brief report: The effects of equine-assisted activities on the social functioning in children and adolescents with autism spectrum disorder. Journal of Autism and Developmental Disorders, 46(10), 3344-3352. doi: 10.1007/ s10803-016-2869-3.

Andrews, L., Attwood, T. \& Sofronoff, K. (2013). Increasing the appropriate demonstration of affectionate behavior, in children with asperger syndrome, high functioning autism, and PDD-NOS: A randomized controlled trial. Research in Autism Spectrum Disorders, 7(12), 1568-1578. doi: https://doi.org/10.1016/j.rasd.2013.09.010.

Argott, P., Townsend, D., Sturmey, P. \& Poulson, C. (2008). Increasing the use of empathic statements in the presence of a non-verbal affective stimulus in adolescents with autism. Research in Autism Spectrum Disorders, 2(2), 341-352. doi: https:// doi.org/10.1016/j.rasd.2007.08.004.

Artigas-Pallares, J. \& Paula, I. (2012). El autismo 70 años después de Leo Kanner y Hans Asperger. Revista de la Asociación Española de Neuropsiquiatría, 32(115), 567-587. doi: http://doi.org/10.4321/S0211-57352012000300008 
Asociación Americana de Psiquiatría (APA) (2014). Manual diagnóstico y estadístico de los trastornos mentales (5 $5^{\text {a }}$ edición). Arlington, Estados Unidos: Editorial Médica Panamericana.

Baghdadli, A., Brisot-Dubois, J., Picot, M. \& Michelon, C. (2010). Comparaison de l'effet de deux interventions prosociales sur l'évolution des capacités d'identification des expressions faciales et du raisonnement social d'enfants avec un syndrome d'asperger ou autisme de haut niveau. Neuropsychiatrie de l'Enfance et de l'Adolescence, 58(8), 456-462. doi: https://doi.org/10.1016/j.neurenf.2010.06.009.

Becker, J., Rogers, E. \& Burrows, B. (2017). Animal-assisted social skills training for children with autism spectrum disorders. Anthrozoös, 30(2), 307-326. doi: 10.1080/08927936.2017.1311055.

Begeer, S., Gevers, C., Clifford, P., Verhoeve, M., Kat, K., Hoddenbach, E. \& Boer, F. (2010). Theory of mind training in children with autism: A randomized controlled trial. Journal of Autism and Developmental Disorders, 41(8), 997-1006. doi: 10.1007/ s10803-010-1121-9.

Belloch, A., Sandín, B. \& Ramos, F. (2009). Manual de Psicopatología (edición revisada). Vol. 2. Madrid, España: McGraw Hill Interamericana.

Chambless, D. L., Sanderson, W. C., Shoham, V., Bennett, Johnson, S., Pope, K. S., CritsCristoph, P., Baker, M., Johnson, B., Woody, S. R., Sue, S., Beutler, L., Williams, D.A. \& McCurry, S. (1996). An update on empirically validated therapies. The Clinical Psychologist, 49(2), 5-18.

Chen, C., Lee, I. \& Lin, L. (2016). Augmented reality-based video-modeling storybook of nonverbal facial cues for children with autism spectrum disorder to improve their perceptions and judgments of facial expressions and emotions. Computers in Human Behavior, 55, 477-485. doi: 10.1016/j.chb.2015.09.033.

Chung, U., Han, D., Shin, Y. \& Renshaw, P. (2016). A prosocial online game for social cognition training in adolescents with high-functioning autism: An fMRI study. Neuropsychiatric Disease and Treatment, 12, 651-660. doi: 10.2147/NDT.S94669.

Cooley, M., Veldorale-Griffin, A., Petren, R. \& Mullis, A. (2014). Parent-child interaction therapy: A meta-analysis of child behavior outcomes and parent stress. Journal of Family Social Work, 17(3), 191-208. doi: 10.1080/10522158.2014.888696

Corbett, B. (2003). Video modeling: A window into the world of autism. The Behavior Analyst Today, 4(3), 367-377. doi: http://dx.doi.org/10.1037/h0100025

Cook, A., Ogden, J. \& Winstone, N. (2018). The impact of a school-based musical contact intervention on prosocial attitudes, emotions and behaviours: A pilot trial with autistic and neurotypical children. Autism, 00(0), 1-10. doi: 10.1177/1362361318787793.

Crozier, S. \& Tincani, M. (2006). Effects of social stories on prosocial behavior of preschool children with autism spectrum disorders. Journal of Autism and Developmental Disorders, 37(9), 1803-1814. doi: 10.1007/s10803-006-0315-7.

Crutchfield, S., Mason, R., Chambers, A., Wills, H. \& Mason, B. (2015). Use of a selfmonitoring application to reduce stereotypic behavior in adolescents with autism: A preliminary investigation of I-Connect. Journal of Autism and Developmental Disorders, 45(5), 1146-1155. doi: 10.1007/s10803-014-2272-x.

Di Renzo, M., Di Castelbianco, F., Petrillo, M., Racinaro, L. \& Rea, M. (2015). Assessment of a long-term developmental relationship-based approach in children with 
autism spectrum disorder. Psychological Reports, 117(1), 26-49. doi: 10.2466/15.10. PR0.117c15z8.

Etchepareborda, M. C. (2005). Funciones ejecutivas y autismo. Revista de Neurología, 40(Supl 1), 155-162.

Gantman, A., Kapp, S., Orenski, K. \& Laugeson, E. (2012). Social skills training for young adults with high-functioning autism spectrum disorders: A randomized controlled pilot study. Journal of Autism and Developmental Disorders, 42(6), 1094-1103. doi: 10.1007/s10803-011-1350-6.

Gevers, C., Clifford, P., Mager, M. \& Boer, F. (2006). Brief report: A theory-of-mindbased social-cognition training program for school-aged children with pervasive developmental disorders: An open study of its effectiveness. Journal of Autism and Developmental Disorders, 36(4), 567-571. doi: 10.1007/s10803-006-0095-0

Goldingay, S., Stagnitti, K., Sheppard, L., McGillivray, J., McLean, B. \& Pepin, G. (2015). An intervention to improve social participation for adolescents with autism spectrum disorder: Pilot study. Developmental Neurorehabilitation, 18(2), 122-130. doi: 10.3109/17518423.2013.855275.

Gresham, F., Sugai, G. \& Horner, R. (2001). Interpreting outcomes of social skills training for students with high-incidence disabilities. Exceptional Children, 67(3), 331-344. https://doi.org/10.1177/001440290106700303

Guivarch, J., Murdymootoo, V., Elissalde, S., Salle-Collemiche, X., Tardieu, S., Jouve, E. \& Poinso, F. (2017). Impact of an implicit social skills training group in children with autism spectrum disorder without intellectual disability: A before-and-after study. PloS ONE, 12(7), 1-18. doi: 10.1371/journal.pone.0181159.

Halle, S., Ninness, C., Ninness, S. \& Lawson, D. (2016). Teaching social skills to students with autism: A video modeling social stories approach. Behavior and Social Issues, 25, 42-53. doi: https://doi.org/10.5210/bsi.v25i0.6190.

Henning, B., Cordier, R., Wilkes-Gillan, S. \& Falkmer, T. (2016). A pilot play-based intervention to improve the social play interactions of children with autism spectrum disorder and their typically developing playmates. Australian Occupational Therapy Journal, 63(4), 223-232. doi: 10.1111/1440-1630.12285.

Jenkins, S. \& Reed, F. (2013). An experimental analysis of the effects of therapeutic horseback riding on the behavior of children with autism. Research in Autism Spectrum Disorders, 7(6), 721-740. doi: 10.1016/j.rasd.2013.02.008.

Kamps, D., Thiemann-Bourque, K., Heitzman-Powell, L., Schwartz, I., Rosenberg, N., Mason, R. \& Cox, S. (2015). A comprehensive peer network intervention to improve social communication of children with autism spectrum disorders: A randomized trial in kindergarten and first grade. Journal of Autism and Developmental Disorders, 45(6), 1809-1824. doi: 10.1007/s10803-014-2340-2.

Kjellmer, L., Hedvall, Å., Fernell, E., Gillberg, C. \& Norrelgen, F. (2012). Language and communication skills in preschool children with autism spectrum disorders: Contribution of cognition, severity of autism symptoms, and adaptive functioning to the variability. Research in Developmental Disabilities, 33(1), 172-180. doi: 10.1016/j. ridd.2011.09.003. 
Koch, S., Mehl, L., Sobanski, E., Sieber, M. \& Fuchs, T. (2015). Fixing the mirrors: A feasibility study of the effects of dance movement therapy on young adults with autism spectrum disorder. Autism, 19(3), 338-350. doi: 10.1177/1362361314522353.

Koegel, L., Ashbaugh, K., Navab, A. \& Koegel, R. (2016). Improving empathic communication skills in adults with autism spectrum disorder. Journal of Autism and Developmental Disorders, 46(3), 921-933. doi: 10.1007/s10803-015-2633-0.

Koehne, S., Behrends, A., Fairhurst, M. \& Dziobek, I. (2016). Fostering social cognition through an imitation-and synchronization-based dance/movement intervention in adults with autism spectrum disorder: A controlled proof-of-concept study. Psychotherapy and Psychosomatics, 85(1), 27-35. doi: 10.1159/000441111.

Kroeger, K., Schultz, J. \& Newsom, C. (2006). A comparison of two group-delivered social skills programs for young children with autism. Journal of Autism and Developmental Disorders, 37(5), 808-817. doi: 10.1007/s10803-006-0207-x.

LaCava, P., Rankin, A., Mahlios, E., Cook, K. \& Simpson, R. (2010). A single case design evaluation of a software and tutor intervention addressing emotion recognition and social interaction in four boys with ASD. Autism, 14(3), 161-178. doi: $10.1177 / 1362361310362085$.

Lozano, J. \& Alcaraz, S. (2011). Software educativo para la enseñanza de competencias emocionales en alumnado con trastornos del espectro autista. Educación XXI, 14(2), 189-212. Recuperado de http://revistas.uned.es/index.php/educacionXX1/article/ viewFile/250/206

Martínez, Y., Martí-Vilar, M. y Martínez, S. (2015). Fomento de la interacción social (conducta prosocial): correlatos neurobiológicos en el Trastorno del Espectro Autista. En C. Ortega et al. (Eds.). El mejoramiento humano. Avances, investigaciones y reflexiones éticas y políticas (pp. 319-335). Granada, España: Editorial Comares.

Mastrominico, A., Fuchs, T., Manders, E., Steffinger, L., Hirjak, D., Sieber, M., ... Koch, S. (2018). Effects of dance movement therapy on adult patients with autism spectrum disorder: A randomized controlled trial. Behavioral Sciences, 8(7), 1-18. doi: 10.3390/bs8070061.

McGarry, L. \& Russo, F. (2011). Mirroring in dance/movement therapy: Potential mechanisms behind empathy enhancement. The Arts in Psychotherapy, 38(3), 178-184. doi: 10.1016/j.aip.2011.04.005.

McKenzie, R., Evans, J. \& Handley, S. (2011). Autism and performance on the suppression task: Reasoning, context and complexity. Thinking \& Reasoning, 17(2), 182-196. doi: 10.1080/13546783.2010.549302.

McVey, A., Dolan, B., Willar, K., Pleiss, S., Karst, J., Casnar, C., ... Hecke, A. (2016). A replication and extension of the PEERS for young adults social skills intervention: Examining effects on social skills and social anxiety in young adults with autism spectrum disorder. Journal of Autism and Developmental Disorders, 46(12), 3739-3754. doi: 10.1007/s10803-016-2911-5.

Mulas, F., Ros-Cervera, G., Millá, M. G., Etchepareborda, M. C., Abad, L. \& Téllez de Meneses, M. (2010). Modelos de intervención en niños con autismo. Revista de Neurología, 50 (Supl 3), 77-84. 
O’Haire, M., McKenzie, S., Beck, A. \& Slaughter, V. (2013). Social behaviors increase in children with autism in the presence of animals compared to toys. PloS ONE, 8(2), 1-10. doi: 10.1371/journal.pone.0057010.

Rabin, S., Israel-Yaacov, S., Laugeson, E., Mor-Snir, I. \& Golan, O. (2018). A randomized controlled trial evaluating the Hebrew adaptation of the PEERS intervention: Behavioral and questionnaire-based outcomes. Autism Research, 11(8), 1187-1200. doi: 10.1002/aur.1974.

Rosenbloom, R., Mason, R., Wills, H. \& Mason, B. (2016). Technology delivered selfmonitoring application to promote successful inclusion of an elementary student with autism. Assistive Technology, 28(1), 9-16. doi: https://doi.org/10.1080/10400435 .2015.1059384.

Smith, T., Scahill, L., Dawson, G., Guthrie, D., Lord, C., Odom, S., Rogers, S. \& Wagner, A. (2007). Designing research studies on psychosocial interventions in autism. Journal of Autism Development Disorders, 37, 354-366. doi: 10.1007/s10803-006-0173-3

Soorya, L., Siper, P., Beck, T., Soffes, S., Halpern, D., Gorenstein, M., ... Buxbaum \& Wang, A. (2015). Randomized comparative trial of a social cognitive skills group for children with autism spectrum disorder. Journal of the American Academy of Child $\mathcal{E}$ Adolescent Psychiatry, 54(3), 208-216. doi: 10.1016/j.jaac.2014.12.005.

Spinrad, T. \& Gal, D. (2018). Fostering prosocial behavior and empathy in young children. Current Opinion in Psychology, 20, 40-44. doi: 10.1016/j.copsyc.2017.08.004.

Stevenson, C., Krantz, P. \& McClannahan, L. (2000). Social interaction skills for children with autism: A script-fading procedure for nonreaders. Behavioral Interventions, 15(1), 1-20. doi: https://doi.org/10.1002/(SICI)1099-078X(200001/03)15:1<1::AIDBIN41>3.0.CO;2-V

Sung, C., Connor, A., Chen, J., Lin, C., Kuo, H. \& Chun, J. (2018). Development, feasibility, and preliminary efficacy of an employment-related social skills intervention for young adults with high-functioning autism. Autism, 00(0), 1-12. doi: https://doi. org $/ 10.1177 / 1362361318801345$.

Tsui, G. \& Rutherford, M. (2014). Video self-modeling is an effective intervention for an adult with autism. Case Reports in Neurological Medicine, 2014, 1-7. doi: $10.1155 / 2014 / 425897$.

Tsunemi, K., Tamura, A., Ogawa, S., Isomura, T., Ito, H., Ida, M. \& Masataka, N. (2014). Intensive exposure to narrative in story books as a possibly effective treatment of social perspective-taking in schoolchildren with autism. Frontiers in Psychology, 5(2), 1-8. doi: 10.3389/fpsyg.2014.00002.

Urrútia, G. \& Bonfill, X. (2010). Declaración PRISMA: Una propuesta para mejorar la publicación de revisiones sistemáticas y metaanálisis. Medicina Clínica (Barcelona), 135(11), 507-511. doi: 10.1016/j.medcli.2010.01.015.

Vesperini, S., Askenazy, F., Renaudo, N., Staccini, P., Hun, S., Legall, E. \& Serret, S. (2015). Intervention pro-sociale chez l'enfant et l'adolescent avec trouble du spectre autistique sans déficit intellectuel: intérêt du support vidéo. L'Encéphale, 41(1), 47-55. doi: 10.1016/j.encep.2013.04.015.

Weinmann, S., Schwarzbach, C., Begemann, M., Roll, S., Vauth, C., Willich, S. N. \& Greiner, W. (2009). Behavioural and skill-based early interventions in children with 
autism spectrum disorders. GMS Health Technology Assessment, 5, 1-10. doi: 10.3205/ hta000072

Yoo, G. \& Kim, S. (2018). Dyadic drum playing and social skills: Implications for rhythm-mediated intervention for children with autism spectrum disorder. Journal of Music Therapy, 55(3), 340-375. doi: https://doi.org/10.1093/jmt/thy013.

Zlomke, K., Jeter, K. \& Murphy, J. (2017). Open-trial pilot of parent-child interaction therapy for children with autism spectrum disorder. Child \& Family Behavior Therapy, 39(1), 1-18. doi: 10.1080/07317107.2016.1267999.

Fecha de recepción: 9 de septiembre de 2019.

Fecha de revisión: 25 de octubre de 2019.

Fecha de aceptación: 25 de enero de 2020. 
